\title{
Viability of Amnion-Chorion membrane with the trophoblast layer in augmented posterior extraction sockets
}

\author{
Mohamed A Maksoud DMD ${ }^{1 *}$ and Amr Fawzy DMD ${ }^{2}$ \\ ${ }^{1}$ Oral Medicine Infection and Immunity, Harvard University School of Dental Medicine, USA \\ ${ }^{2}$ Private Practice, Weymouth, Ma, USA
}

\author{
*Corresponding author: Mohamed A Maksoud DMD, Oral Medicine Infection and \\ Immunity, Harvard University School of Dental Medicine, 188 Longwood Avenue, Boston, \\ Ma. 02118, USA.
}

Received Date: October 08, 2021

Published Date: October 21, 2021

\begin{abstract}
Augmentation of the extraction sockets with bone and a membrane prior to dental implants insertion has been an integral part of the dental practice due to the advantages of providing adequate hard and soft tissue support essential for the implant emergence profile. Several bone graft materials and membranes have been recommended for the socket preservation however most of the commercially available resorbable membranes dissolve prematurely when exposed resulting in loss of the graft material. With the introduction of the human placental membranes into the dental field expedited wound healing was demonstrated even when exposed due to the abundance of growth factors. In this study Amniotic membrane which processed in a way to preserve the trophoblast layer has been tested on top of augmented extraction sockets. Clinically this membrane was easier to handle due to the increased thickness as a result of combining amnion chorion and trophoblast layer, it has also shown expedited healing through tissue formation on top of the bone graft mass as soon as two weeks. The histological examination demonstrated a considerable layer of fully matured epithelized tissue covering the bone in a short period of time. The results confirmed the viability of the Amnion Chorion and trophoblast membrane in generating epithelized tissue is a short period of time when used in covering bone grafts in extraction sockets.
\end{abstract}

\section{Introduction}

The human placental membrane is made of two layers amnion and chorion. The amnion layer has five layers and some growth factors [1], clinically it's difficult to apply by itself for grafting due to lack of handling and possible migration from the wound during healing [2]. On the other hand the chorion is made of four layers cellular, reticular, pseudomembranous and trophoblast [1]. While the commercially available amnion chorion membrane does only include the amnion and chorion with the exception of the trophoblast layer which is separated during the processing, it has shown promising results in intra-oral grafting [3] however lacked handling properties during grafting due to its thinness. A newly introduced membrane Amnio Excite *which will be referred to as dehydrated human amnio chorion membrane with trophoblast dHACMT does include the trophoblast layer which is thicker than the chorion layer (Figure 1) has shown great handling properties due to its thickness and proven to be a great barrier by preventing fluids as well as bacterial ingress [4]. In this study the dHACMT membrane will be tested in grafted extraction sockets covering the bone mass with its ability to generate gingival tissue. Additionally a histological examination will be rendered to determine the nature of the healed tissue components.

\section{Materials and Methods}

Fifteen patients selected with planned posterior extraction and dental implant treatment to follow. They were all enrolled and prepared for surgery in accordance with accepted dental practice guidelines in a private practice setting. The selected sites planned for non-surgical atraumatic extraction with at least three 
remaining walls. The patients were seen first for evaluation and written consent signing explaining the procedure including risk factors and preoperative photographs. All patients operated on under local anesthesia (2\% Xylocaine with epinephrine 1:100,000) and traumatic extraction rendered with socket degranulation to ensure clearance from foreign materials or granulation tissue. Socket augmented in a non-compact manner using a bone graft mass consisting of cortico-cancellous allograft $\bullet \bullet$ after hydration using sterile water and covered with the membrane and tucked $2-3 \mathrm{~mm}$ below the buccal and lingual flaps. Sutures rendered using 4-0 polytetrafluorethylene suture leaving the membrane exposed.
Postoperative instructions discussed in addition to ice pack. Postoperative antibiotics orally administered after surgery; $2 \mathrm{gm}$ of Amoxicillin daily for one week in addition to pain relief medication. Postoperative intra-oral photographs taken of the site. All patients monitored in two, and six weeks for the healing of the extraction socket specifically for the soft tissue coverage on top of the bone mass. At the sixth week visit one case was selected for a soft tissue biopsy during the procedure of implant insertion.

-• Salvin Dental Specialties, 3450 Latrobe Dr, Charlotte, NC 28211

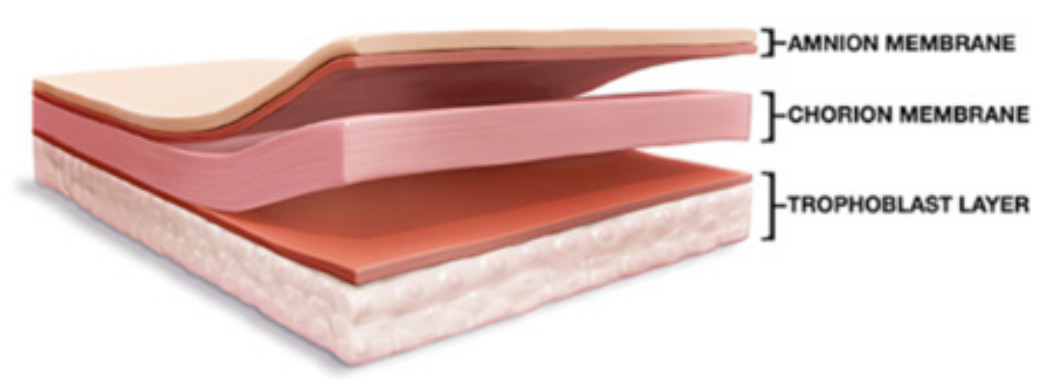

Figure 1: Showing a diagram illustrating the three layers of Amnion, chorion and trophoblast.

\section{Results}

The surgical sites showed uneventful healing with no complications on the second week of the suture removal visit demonstrating initial coverage of the bone graft mass with a healing tissue on top and no loss of the bone graft material (Figures 2). On the sixth week fully formed epithelized tissue took place on top of the extraction socket which resembles the patient's native keratinized gingival tissue (Figures 3). All cases showed no visible loss of the bone graft material during the or the membrane during the healing, no other complications were reported. A periapical film taken at the sixth week visit and compared to the pre-operative film revealed good bone fill and readiness of the site to receive dental implant ( Figures 4-6).

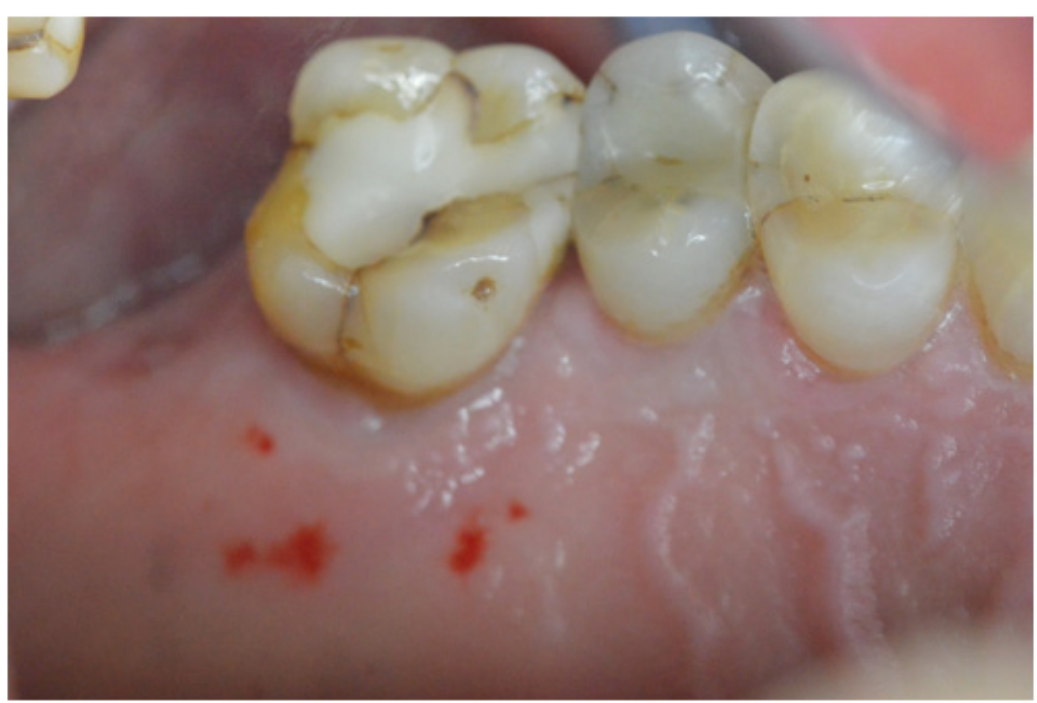

Figure 2: Showing before the extraction. 


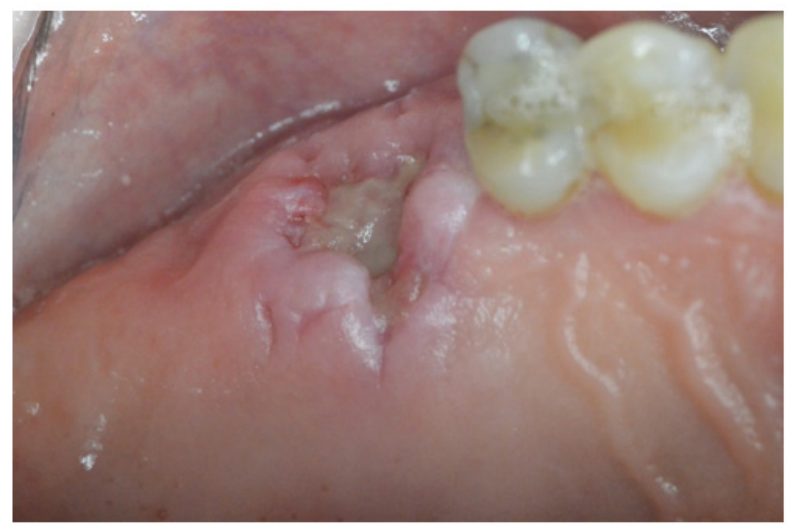

Figure 3: Showing two weeks healing with initiated soft tissue formation replacing the membrane.

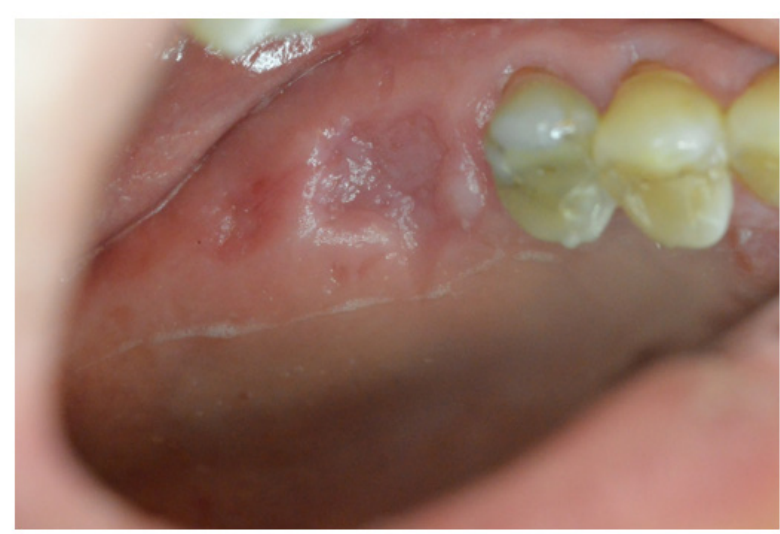

Figure 4: Showing complete maturation of the soft tissue covering into keratinized tissue after six weeks.

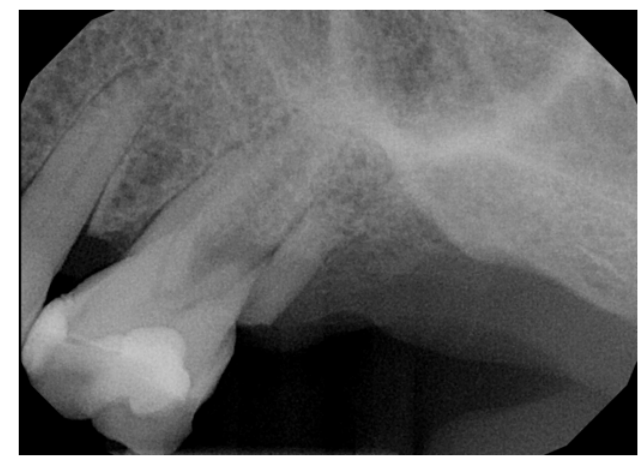

Figure 5: Showing radiograph of the site before the extraction.

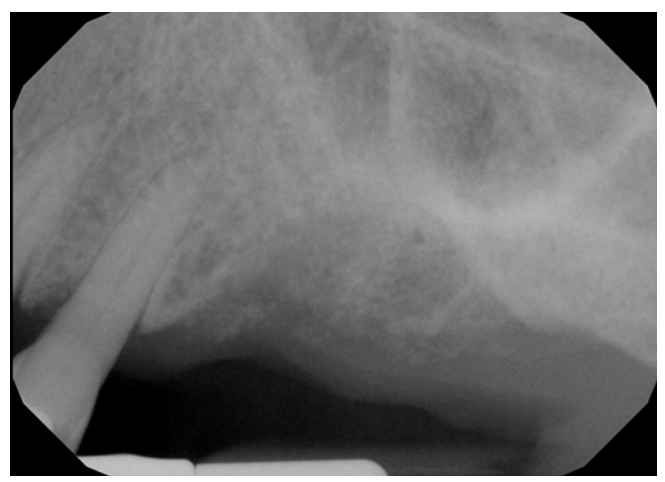

Figure 6: Showing radiograph of the site six weeks after the extraction and bone grafting. 


\section{The Histological Examination}

The histology revealed keratinized stratified squamous epithelium and supporting connective tissue. The surface of the epithelium was covered by a thickened layer of ortho and para keratin. The spinous cell layer variably thickened and formed numerous elongated and bulbous rete ridges. The basal cell layer was intact and the underlying connective tissue was composed of dense bundles of collagen fibers interspersed by fibroblasts and capillaries (Figure 7).

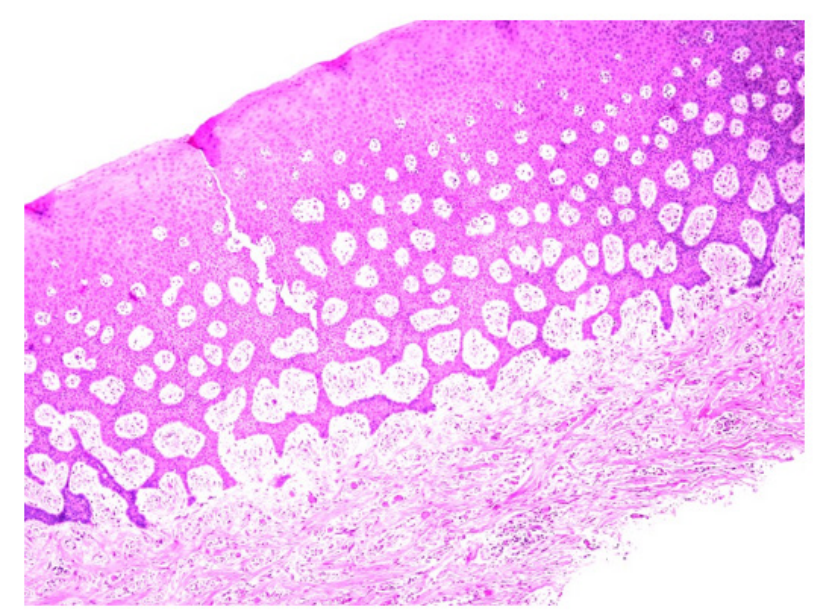

Figures 7: Showing histological slide of the keratinized tissue in six weeks with a thick layer of hyperkeratosis.

\section{Discussion}

Unlike the Amnion chorion membrane which lacks the trophoblast layer dHACMT provides a thick base for better handling and wound stability. In addition, the trophoblast layer contains the majority of the biological factors in the whole placental membrane [5]. When the trophoblast layer is removed many endogenous proteins are lost including fibronectin. In a human study fibronectin was shown to be an integral part of cell migration and attachment to extracellular matrix during the healing process $[6,7]$. In a previous study by the first author [3] clinical comparable results were noticed when Amnion chorion was used however no histological study was rendered. In this study the confirmation of the histological evidence that keratinized tissue was formed was mainly due to the abundance of growth factors in the trophoblast layer. In a histological study to determine the viability of the membrane it was determined that it has preserved its extracellular matrix and bioactivity during the wound healing, this was mainly due to the array of its cytokines that are known to regulate the wound healing process [8]. Additionally a similar study by the same first author and others confirmed the membrane possessed several distinct physiological processes the ultimately signals and maintains the wound healing process cell proliferation, metalloproteinase activity, inflammation and progenitor cell proliferation [9].

\section{Conclusion}

The clinical observation of all cases confirmed biological acceptance of the dHACMT into the surgical site and its viability in covering the bone mass with no loss as early as two weeks. The process of healing continued with the formation of fully matured keratinized tissue. The histological examination revealed significant cellular activity in the surgical wound that have resulted in a fully keratinized tissue. The results of the current study can confirm the viability of the dHACMT in expediting healing in augmented extraction sockets including rapid epithelization.

\section{Acknowledgment}

The author would like to thank Salvin Dental for their contribution.

\section{Conflict of Interest}

Additionally the author states no conflict of interest in relation to the above manuscript.

\section{References}

1. Bourne G (1962) The foetal membranes. A review of the anatomy of normal amnion and chorion and some aspects of their function. Postgrad Med J 38: 193-201.

2. Murphy SV, Skardal A, Nelson RA, Sunnon K, Reid T, et al. (2020) Amnion membrane hydrogel and amnion membrane powder accelerate wound healing in a full thickness porcine skin wound model. Stem Cells Transl Med 9(1): 80-92.

3. Maksoud M, Guze K (2018) Tissue expansion of dental extraction sockets using dehydrated human amnion/chorion membrane: Case series. Clin Adv Periodontics 8: 111-114.

4. Verbruggen SW, Oyen ML, Phillips AT, Nowlan NC (2017) Function and failure of the fetal membrane: Modelling the mechanics of the chorion and amnion. PLoS One 12(3): e0171588.

5. LifeNet-Health (2020) TR-004-2020 Characterization of the Amnion, Chorion, and Trophoblast Layers of Decellularized and Freeze-Dried Placental Membrane.

6. Hsiao CT, Cheng HW, Huang CM, Li HR, Ou MH, et al. (2017) Fibronectin in cell adhesionand migration via $\mathrm{N}$-glycosylation. Oncotarget 8(41): 70653-70668. 
7. Schultz GS, Wysocki A (2009) Interactions between extracellular matrix and growth factors in wound healing. Wound Repair Regen 17(2): 153162.

8. Koob TJ, Lim JJ, Massee M, Zabek N, Denozière G (2014) Properties of dehydrated human amnion/chorion composite grafts: implications for wound repair and soft tissue regeneration. J Biomed Mater Res B Appl Biomater 102: 1353-1362.
9. Koob TJ, Rennert R, Zabek N, Michelle Massee, Jeremy J Lim, et al. (2013) Biological properties of dehydrated human amnion/chorion composite graft: implications for chronic wound healing. Int Wound J 10: 493-500. 